\title{
The Serological Gastric Biopsy: A Non-endoscopical/Histopathologic Diagnostic Approach in Management of the Dyspeptic Patients
}

\author{
${ }^{1}$ Mahmoud R.A.K., ${ }^{2}$ Morcos H.H., ${ }^{3}$ Hegazi A.A., ${ }^{4}$ Abo Seif M.A. and ${ }^{4}$ El-Hadidy K.S. \\ ${ }^{1}$ Medical Biochemistry and ${ }^{4}$ Internal Medicine Departments, Faculty of Medicine \\ Cairo University, Egypt \\ ${ }^{2}$ Microbiology and ${ }^{3}$ Pathology Departments: National Hepatology and Tropical Medicine Research \\ Institute, Cairo, Egypt
}

\begin{abstract}
Objective: To asses the sensitivity, specificity and the predictive value of a 'gastropanel' blood test, including serum pepsinogen I (S-PGI) and amidated gastrin-17 (S-G-17) in the diagnosis of atrophic gastritis most probably due to helicobacter pylori (Hp) and to compare the results of serology with the endoscopic/histopathologic findings as a gold standard diagnostic tool. This study comprised 86 dyspeptic outpatients who underwent a diagnostic gastroscopy with multiple biopsies from the antrum and corpus for histopathologic examination. Histopathologically, the series of cases included 46 patients diagnosed as having atrophic gastritis. Twenty five had an antral atrophy, 6 had corpus atrophy and 15 patients had an atrophic gastritis in both the antrum and corpus (multifocal atrophic gastritis) MAG. The remaining 40 cases were considered as controls, $32 \mathrm{had}$ a non-atrophic gastritis (NAG) and both the antrum and corpus were normal and healthy in 8 subjects. The fasting levels of SPGI and antibodies to Hp IgG-antibodies (S-HpAb) and postprandial amidated S-G-17 (S-G-17prand) were measured $20 \mathrm{~min}$ after a protein-rich drink and were determined using enzyme immunoassay (EIA) methods. The serologic and morphologic results were compared with estimating the sensitivity, specificity, positive and negative predictive (PPV and NPV) and accuracy values of the tests (S-G-17 and S-PGI). Mean values of both S-G-17prand and S-PGI decreased with increasing grade of antral and corpus atrophy, respectively. A low S-PGI $\left(<25 \mu \mathrm{g} \mathrm{L}^{-1}\right)$ was found in 4 of 6 patients with corpus atrophy $(66.70 \%)$ and in 5 of $32(15.62 \%)$ patients with NAG in the biopsy specimens. A low S-G17 prand $\left.(<5 \text { pmol L})^{-1}\right)$ was found in 13 patients with $\mathrm{Hp}$-associated antral atrophy and in 5 of 32 $(15.62 \%)$ patients with NAG. The diagnosis of gastritis obtained with the blood test panel is in good agreement with the endoscopic and biopsy findings: $92 \%$ of the patients went into same gastritis categories with both the panel and endoscopic/histopathologic findings. The sensitivity, specificity, PPV, NPV and accuracy values of the blood test panel (S-G-17prand and S-PGI) in delineation of patients with atrophic gastritis (either in the antrum or the corpus, or both) versus NAG and normal stomach were $87.5,82.60,81.40,88.40$ and $85 \%$ respectively. Low levels of S-G-17prand and S-PGI are conceivable biomarkers of atrophic gastritis. The serologic method gave a reasonable sensitivity, specificity, PPV, NPV and accuracy values in the diagnosis of atrophic gastritis. So, it may be offered as the screening tool for atrophic gastritis.
\end{abstract}

Key words: Atrophic gastritis, endoscopic/histopathologic, serum Helicobacter pylori, pepsinogen I

\section{INTRODUCTION}

Helicobacter pylori $(\mathrm{Hp})$-infection is one of the most common chronic infections worldwide. Half of the global population is infected by Hp. Most infected patients will suffer from chronic gastritis and over $20 \%$ of those may suffer from peptic ulcer. In nearly half of the infected cases the gastritis develops over the years into atrophic gastritis, which increases considerably the risk of gastric cancer ${ }^{[1]}$. The World Health Organization (WHO) considered $\mathrm{Hp}$ as a class I carcinogen ${ }^{[2]}$. Correa $^{[3]}$ proposed the consecutive events leading to gastric cancer development: normal mucosa-chronic active gastritis-chronic atrophic gastritis-intestinal metaplasia (IM)-dysplasia-carcinoma in situ. This cascade of changes associates with a trigger role of $\mathrm{Hp}$ is called "Correa's gastric precancerous cascade" ${ }^{[3,4]}$. Atrophic corpus gastritis leads to hypo- or achlorohydria and in advanced cases result in malabsorption of vitamin $\mathrm{B} 12^{[5]}$. Until now, in accordance with the Maastricht 2 (2000)-guidelines, the patients suffering from $\mathrm{Hp}$-infection and dyspepsia have been recommended to be treated ${ }^{[4]}$.

The two main causes that result in distinct topographic types of atrophic gastritis can be distinguished histologically. Hp-associated atrophic gastritis usually multifocal atrophic gastritis (MAG) that involves both the antrum and corpus, is highly 
prevalent in developing countries and Asia and autoimmune gastritis essentially restricted to the gastric corpus is more prevalent in Northern Europe ${ }^{[6]}$.

Atrophic gastritis is usually diagnosed with endoscopy and biopsies from gastric antrum and corpus which is invasive. The breath test and the microscopical examination of $\mathrm{Hp}$ in biopsy samples sometimes produce a negative result. As a result, the $\mathrm{Hp}$-infection causing atrophic gastritis remains untreated. Evenmore, Hp test alone has limited value, because it only diagnoses the presence or absence of the Hp bacterium in the gastric mucosa. Very important information is still missing, namely the damage-known as atrophic gastritis $^{[7]}$. However, atrophic gastritis of the gastric corpus and fundus can be diagnosed nonendoscopically by assaying the levels of S-PGI, or the ratio of $\mathrm{S}$-PGI to S-PGII or total gastrin ${ }^{[8]}$. With worsening of the corpus atrophy (loss of normal oxyntic glands) the S-PGI tends to decrease. The gastrin secreted by the antrum is over $90 \%$ of type G-17 which increases after the dietary stimulus ${ }^{[9]}$ In atrophic antral gastritis, the loss of antral glands results in a decrease in the number of antral $\mathrm{G}$ cells ${ }^{[10-12]}$. Recent evidence has shown that by adding new assays (S-G-17 and S-PGI as biomarkers of gastric antral and corpus mucosa respectively) to the $\mathrm{Hp}$ assay, one can determine from a single blood sample the presence, severity and location of atrophic gastritis ${ }^{[9-13]}$.

The aim of the present work was to study whether patients with atrophic gastritis associated with chronic Hp infection in Egyptian dyspeptic patients can be diagnosed and typed non-endoscopically if the serum levels of pepsinogen I (S-PGI) and gastrin-17 (S-G-17) are assayed in connection with Helicobacter pylori IgGantibodies testing for preventing further development of gastric cancer.

Subjects and Methods: This study comprised 86 adult outpatients who underwent diagnostic uppergastrointestinal endoscopy for various dyspeptic symptoms, recurrent abdominal pain and vomiting in the Internal Medicine clinics of Beni-Souief and Kasr Al Aini hospitals, Faculty of Medicine, Cairo University. The mean age of patients was 55 years (range:30-75 years) and the male to female ratio was 48:38. Patients were asked to stop treatment affecting the acid output such as antacids or proton pump inhibitors one week before the laboratory tests which were done at least one week after endoscopy as gastroscopy may also change the gastrin output. Exclusion criteria included the following: allergic reactions to protein drink e.g. allergic for egg, soy, chocolate or milk products, history of malignancy, gastrectomy or debilitating medical illness (severe cardiovascular or pulmonary disease, chronic renal failure). Gastric biopsies were taken from antrum and corpus, at least 2 from each site, in addition to biopsies from endoscopically visible mucosal lesions for histologic assessment.

Endoscopy and histopathology: the biopsy specimens were fixed in $10 \%$ buffered formalin in room temperature and then embedded in paraffin blocks. Histological Sections were stained using hematoxylin and eosin for routine examination and modified Giemsa for detection of Hp. With histological examination (Fig. 1-3): the presence of $\mathrm{Hp}$ organisms, follicular gastritis, the nature of inflammation (acute or chronic), activity, mucosal atrophy, lymphoid follicles, goblet cells and intestinal metaplasia (IM)/dysplasia were noted and graded according to the updated Sydney System ${ }^{[14]}$. Cases of chronic follicular gastritis were subjected to indirect immunohistochemical staining technique using standard Streptavidin-Biotin method and monoclonal antibody for CD20 ${ }^{[15]}$ (Dako LSAB2 system, USA).

The patients were classified into the following groups: normal and healthy mucosa: no gastritis, no atrophy; NAG: presence of gastritis without biopsy evidence of atrophy or IM, this category was not subdivided into antral or corpus gastritis; lastly atrophic gastritis (loss of normal glands by at least half) in antrum, corpus or both (MAG): which were subdivided for the first two into mild A1, moderate A2 or severe A3. IM is also an indicator of atrophy, which suggests the loss of glands and atrophy.

Serological tests: the basal blood samples for measurements of S-PGI and S-HpAb were drawn after an overnight fast. The sample for postprand amidated gastrin-17 (G-17prand) was taken 20 minutes after a protein drink (10 g chocolate flavored soy protein powder foil bag dissolved in $100 \mathrm{ml}$ water, Biohit Plc, Helsinki, Finland). Due to the sensitivity of S-G-17 (easily destroyed), it is recommended that coagulation of tubes for serum must not exceed $30 \mathrm{~min}$ at room temperature $\left(20-25{ }^{\circ} \mathrm{C}\right)$. Otherwise, at warmer temperatures the blood tubes for serum are placed into crushed ice bath for coagulation that should not exceed 60 min. Serum after clotting is separated by centrifugation at $2000 \mathrm{~g}$ for $15 \mathrm{~min}$. Serum was aliquoted and stored frozen at $-70^{\circ} \mathrm{C}$ until assayed. Once the samples were unfrozen, we kept them in an ice water bath during pipeting steps. S-PGI and S-G-17 were assayed with EIA methods using highly specific monoclonal antibodies to $\mathrm{PGI}^{[16]}$ and amidated G-17 ${ }^{[17]}$ respectively and $\mathrm{Hp} \mathrm{IgG}$ antibodies ${ }^{[1]}$ were determined using a polyclonal EIA method with Biohit GastroPanel ${ }$ (Biohit Plc, Helsinki, Finland) according to the manual protocols.

Diagnosis of atrophic gastritis by the blood test panel: According to the instructions of the manufacturer and various literatures ${ }^{[10,11,13,18,21]}$ a decision tree (algorithm) with an empirical cut-off with best discrimination can be constructed for the test panel to depict patients with different phenotypes of gastritis. 
This decision tree begins with the consideration whether the patient has $\mathrm{Hp}$ infection with consequent gastritis (S-HpAb IgG titers: > 30 enzyme immunoassay unit (EIU)-positive result). Levels of SPG1 $<25 \mu \mathrm{g} \mathrm{L}^{-1}$ were estimated as markers of corpus atrophy, levels of S-G-17prand $<5$ pmol $\mathrm{L}^{-1}$ were estimated as markers of antral atrophy, levels of S-G17prand $<10$ pmol L $^{-1}$ and S-PG1 $<50 \mu \mathrm{g} \mathrm{L}^{-1}$ were estimated as markers of gastric antrum and corpus atrophy.

Statistical analysis: All analyses were performed with the Statistical Package for Social Sciences (SPSS) software (version 11 for Windows). Numerical data were expressed as mean $\pm \mathrm{SD}$, median and range. Comparisons were performed by analysis of variance", ANOVA', on ranks, Scheffe's test was used as a posthoc test. Correlations were tested by Spearman's Chi square test for the correlation between test panel and endoscopy/histopathology findings. Comparisons and correlations were considered statistically significant when $\mathrm{P}<0.05$. The sensitivity, specificity, PPV, NPV and accuracy of the test panel were calculated and compared to the results of endoscopy/histopathology ${ }^{[22]}$.

\section{RESULTS}

Table 1 represented the clinical data of our 86 dyspeptic patients. On the basis of histological appearances in multiple endoscopical biopsies (Fig. 1-3): of the 86 patients, 46 (cases) had an atrophic gastritis, of these 19 had an advanced (moderate or severe) antral atrophy; 5 had advanced (moderate or severe) corpus atrophy and 15 had MAG in both antrum and corpus. The controls comprised of 40 subjects, of whom 32 had NAG and the antrum and corpus were normal and healthy in 8. Histopathologically, there were $74(86 \%) \mathrm{Hp}$ positive (+ve) cases; meanwhile, S$\mathrm{HpAb}$ associated gastritis was present in 72 of 86 (84\%) dyspeptic patients. Among them 29 had evidence of NAG. Gastritis with atrophy was present in 46 subjects, 43 and 42 of them were $\mathrm{S}-\mathrm{HpAb}$ and histopathologically +ve respectively. In 8 of 46 patients with atrophic gastritis we detected IM/dysplasia with villous formation of stomach mucosal epithelium, in whom we revealed decreased levels of S-G-17prand $<5$ pmol $\mathrm{L}^{-1}$ and $\mathrm{S}-\mathrm{PG} 1<25 \mu \mathrm{g} \mathrm{L} \mathrm{L}^{-1}$. Also of whom 2 patients were $\mathrm{S}-\mathrm{HpAb}+\mathrm{ve}$ and -ve in histopathology, otherwise, the remaining 6 patients were $\mathrm{Hp}$ +ve serologically and histopathologically. Gastric erosion was detected in 4 patients ( 2 with NAG and the other 2 with corpus atrophy) and gastric ulcer was detected in 1 patient with moderate degree of antral atrophy. Follicular gastritis was detected in 10 patients (5 with moderate-severe antral atrophy, 1 with severe corpus atrophy, 4 with both corpus and antrum atrophy); Hp was found in 9 patients verified both serologically and histopathologically. Table 2 and Fig. 4:

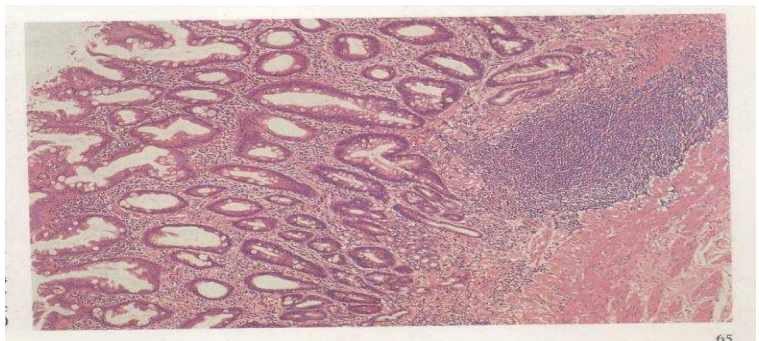

Fig. 1: Chronic atrophic gastritis with complete loss of the parietal and chief cells, the mucosa consists of irregular glands with large numbers of goblet cells and a villous structure of the surface epithelium "Intestinal metaplasia " and the submucosa shows a large lymphoid follicle ( $\mathrm{H} \& \mathrm{E}$ X100 )

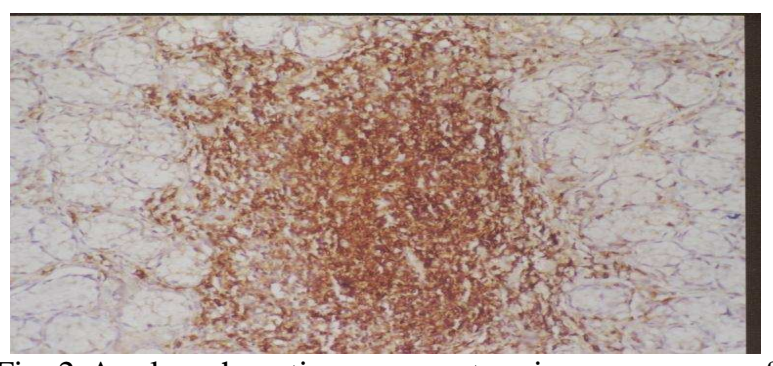

Fig. 2: A lymphocytic aggregate in a case of Helicobacter pylori-associated follicular gastritis. The aggregate is composed of many B cells immunostained with CD20 antibody immunoperoxidase, original magnification $\mathrm{X} 400)$

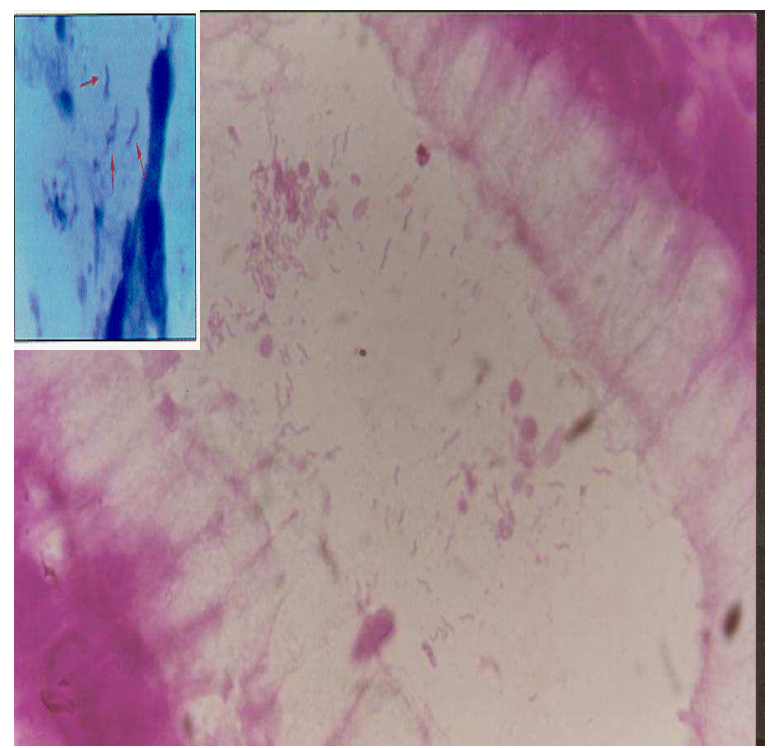

Fig. 3: Gastric mucosa infected by Helicobacter Pylori (Hp) ( H\&E X1000), at the corner Hp organism stained by modified Giemsa stain

showed that the levels of S-G-17prand and S-PGI in antral, in MAG (and S-PGI only in corpus atrophy) were significantly lower $(\mathrm{P}<0.001)$ than those in nonatrophic state. 
Am. J. Immunol., 2(4): 88-96, 2006

Table 1: Characteristic data of dyspeptic patients

\begin{tabular}{|c|c|c|c|c|c|}
\hline Parameters & $\begin{array}{l}\text { Normal } \\
\mathrm{n}=8(9.30 \%)\end{array}$ & $\begin{array}{l}\text { Non-atrophic } \\
\text { Gastritis } n=32 \\
(37.21 \%)\end{array}$ & $\begin{array}{l}\text { Antral atrophy } \\
\mathrm{n}=25(29.07 \%)\end{array}$ & $\begin{array}{l}\text { Corpus atrophy } \\
\mathrm{n}=6(6.98 \%)\end{array}$ & $\begin{array}{l}\text { Multifocal atrophic gastritis } \\
\text { (MAG) Antral + Corpus } \\
\text { atrophy } n=15(17.44 \%)\end{array}$ \\
\hline \multicolumn{6}{|l|}{ Helicobacter pylori } \\
\hline IgG antibodies 14 - & $8(100 \%)$ & $3(9.40 \%)$ & ------ & $3(50 \%)$ & ------ \\
\hline $72+$ & ---- & $29(90.60 \%)$ & $25(100 \%)$ & $3(50 \%)$ & $15(100 \%)$ \\
\hline Histopathology 12 - & $8(100 \%)$ & ----- & $1(4 \%)$ & $2(33.3 \%)$ & $1(6.70 \%)$ \\
\hline $\begin{array}{c}74+ \\
\text { Grades of antral atrophy }\end{array}$ & ------- & $32(100 \%)$ & $24(96 \%)$ & $4(66.70 \%)$ & $14(93.30 \%)$ \\
\hline A1 (mild) & --- & ---- & $6(24 \%)$ & ---- & ----- \\
\hline A2 (moderate) & & & $6(24 \%)$ & & \\
\hline A3 ( severe) & & & $13(52 \%)$ & & \\
\hline \multicolumn{6}{|l|}{ Grades of corpus atrophy } \\
\hline A1 (mild) & & & & $1(16.70 \%)$ & \\
\hline A2 ( moderate) & & & & $2(33.30 \%)$ & \\
\hline A3 ( severe) & & & & $3(50 \%)$ & \\
\hline $\begin{array}{lcr}\text { Intestinal } & \text { metaplasia } & \text { and } \\
\text { dysplasia } & \text { with } & \text { villous } \\
\text { formation } & & \end{array}$ & ----- & ----- & 4 & 1 & 3 \\
\hline Helicobacter $\quad$ pylori & & & $3(+,+)$ & $1(+,+)$ & $2(+,+)$ \\
\hline $\begin{array}{l}\text { Serology,histopathology } \\
\text { Follicular gastritis }\end{array}$ & ------- & -------- & $1(+,-)$ & ---- & $1(+,-)$ \\
\hline Serology,histopathology & & & $5(+,+)$ & $1(+,-)$ & $4(+,+)$ \\
\hline
\end{tabular}

Negative-, Positive +

Table 2: Serum levels of postprandial amidated gastrin-17 (S-G-17 prandial) and pepsinogen I (S-PGI) in dyspeptic patients

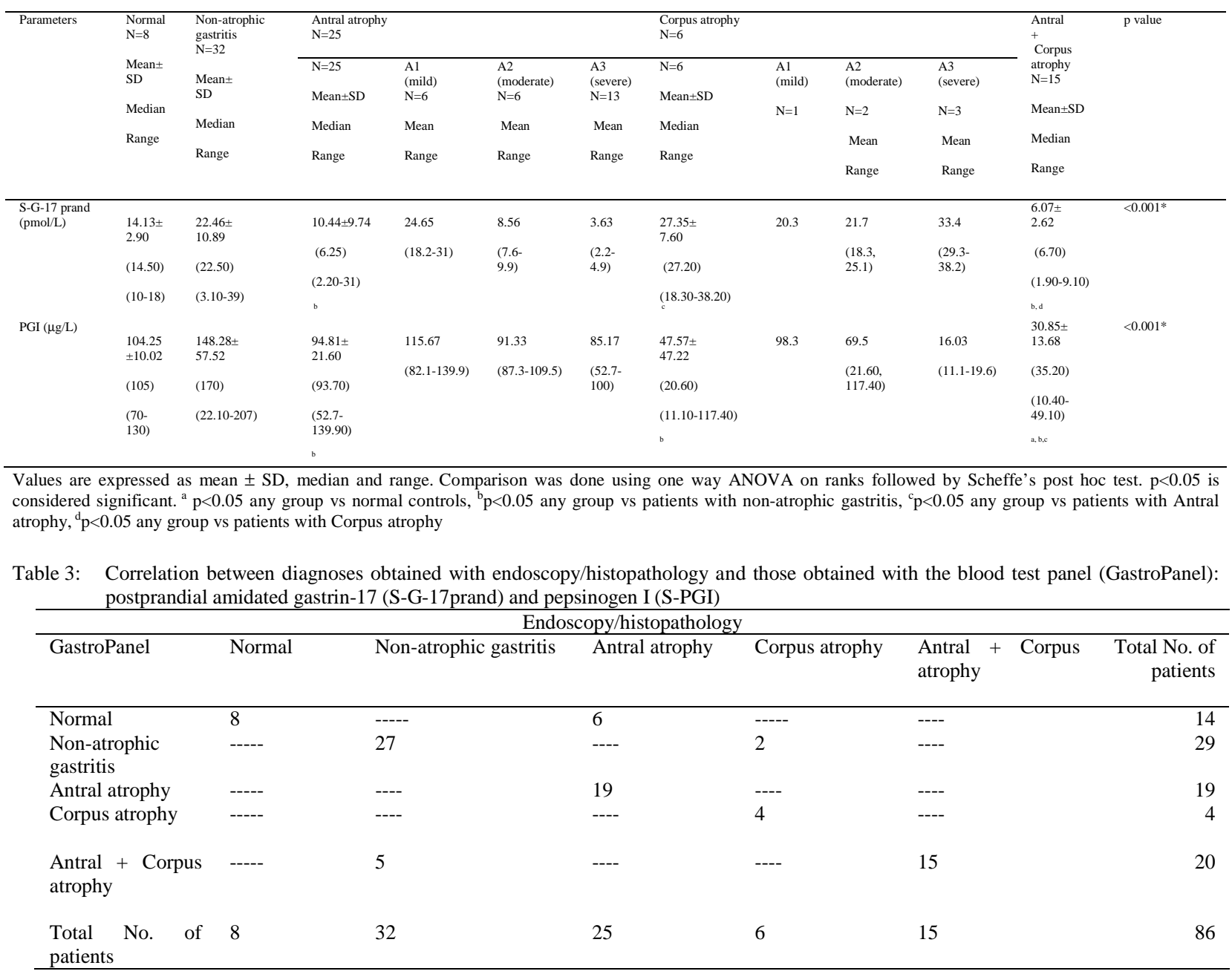


Table 4: Sensitivity, specificity, positive and negative predictive values and accuracy of the blood test panel (GastroPanel): postprandial amidated gastrin-17 (S-G-17 prand) and pepsinogen I (S-PGI) for the detection of atrophic gastritis (antrum, corpus and both antrum and corpus) in 46 dyspeptic patients referred for endoscopy/histopathology. The reference group (40) comprised of normal subjects $(\mathrm{n}=8)$ and patients with non-atrophic gastritis $(\mathrm{n}=32)$. Cut-off was given in parentheses

\begin{tabular}{|c|c|c|c|c|c|}
\hline Diagnosis of atrophic gastritis & $\begin{array}{l}\text { Sensitivity } \\
\%\end{array}$ & $\begin{array}{l}\text { Specificity } \\
\%\end{array}$ & $\begin{array}{l}\text { Positive predictive } \\
\text { value } \%\end{array}$ & $\begin{array}{l}\text { Negative } \\
\text { predictive value } \%\end{array}$ & $\begin{array}{l}\text { Accuracy } \\
\%\end{array}$ \\
\hline $\begin{array}{l}\text { S-G-17 prandial } \\
(<10 \mathrm{pmol} / \mathrm{L})\end{array}$ & 87.50 & 73.90 & 74.50 & 87.10 & 80 \\
\hline S-PGI $(<50 \mu \mathrm{g} / \mathrm{L})$ & 87.50 & 43.50 & 57.40 & 80 & 64 \\
\hline $\mathrm{S}-\mathrm{G}-17 \mathrm{prand}(<10 \mathrm{pmol} / \mathrm{L})$ and $\mathrm{S}-\mathrm{PGI}(<50 \mu \mathrm{g} / \mathrm{L})$ & 87.5 & 82.60 & 81.40 & 88.40 & 85 \\
\hline
\end{tabular}

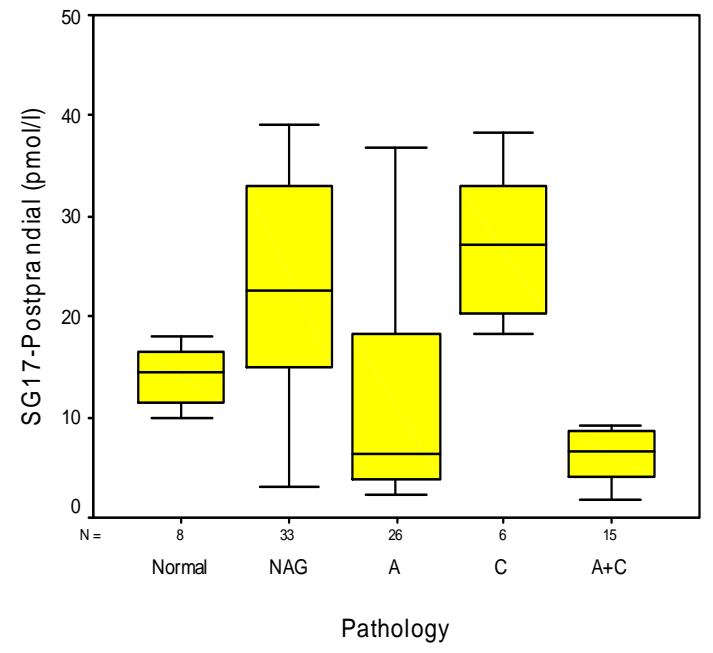

A

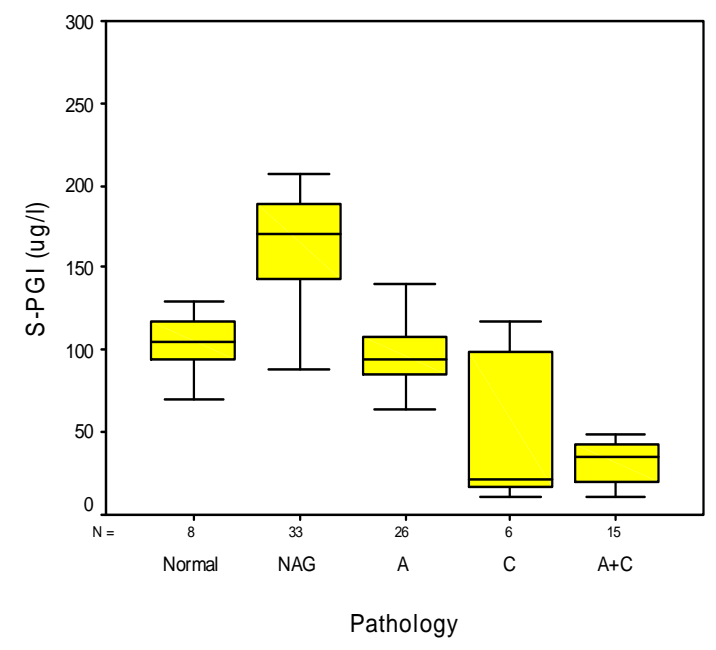

B

Fig. 4: A: Comparison of serum postprandial amidated gastrin-17 (S-G-17 prand) (pmol/L) and B: serum pepsinogen I (S-PGI) (ug/L) levels and morphological status of gastric mucosa. Box and whiskers plot: the box indicated the cases between the $25^{\text {th }}$ and $75^{\text {th }}$ percentiles and the whiskers indicated the largest and smallest observed values. The median value was shown with a horizontal line. NAG: Non-atrophic gastritis, A: Antral atrophy, C: Corpus atrophy, $\mathrm{A}+\mathrm{C}$ : Antral+ Corpus atrophy
Meanwhile, S-G-17prand was significantly increased in those subjects with corpus atrophy in comparison to those with antral atrophy. Mean values of both S-G17prand and S-PGI decreased with increasing grade of antral and corpus atrophy, respectively. All 19 (76\%) of 25 patients with an Hp-positive moderate or severe atrophic antral gastritis had S-G-17prand levels <10 pmol $\mathrm{L}^{-1}$. Of the 5 patients with moderate or severe corpus atrophy in endoscopic biopsies, 4 patients had SPGI levels $<25 \mu \mathrm{g} \mathrm{L}^{-1}$. Table 3 showed the results of the comparison between diagnoses obtained with endoscopy/ histopathology and those obtained with the blood test panel: $92 \%$ of the patients went into same gastritis categories with both the panel and endoscopic/histopathologic findings, (Pearson Chi square test $=230, \mathrm{p}<0.001)$. Table 4: we evaluated sensitivity, specificity, PPV, NPV and accuracy values of discrete test included: S-G-17prand and S-PGI or in combinations by using their cut-off levels with the best discrimination recommended in the algorithm, for delineation of patients with atrophic gastritis in general of any topographical type versus NAG and normal stomach. The sensitivity, specificity, PPV, NPV and accuracy values of the S-G-17prand and S-PGI combined were 87.5, 82.60, 81.40, 88.4 and $85 \%$ respectively, (Pearson Chi square test $=38, \mathrm{p}<0.001$ and measure of agreement: kappa=0.66,p<0.001).

\section{DISCUSSION}

The present study showed that out of 86 dyspeptic patients 25 had an antral atrophy, antrum is the site of Hp growing ${ }^{[23]}, 6$ had corpus atrophy and 15 patients had MAG. We also found that levels of S-G-17prand and S-PGI were significantly lowered in patients with antral and corpus atrophy than those with NAG; furthermore, their levels decreased with increasing grades of atrophy respectively. These findings were in agreement with previous studies ${ }^{[10,11,13,18,20,21,24]}$. In the study of Bodger et al. ${ }^{[24]}$ of 69 dyspeptic patients $41.20 \%$ had Hp-antral atrophy, 52.9\% with MAG and $5.9 \%$ had corpus atrophy in the Hp positive group. Hartleb et al. ${ }^{[21]}$ found that of 55 dyspeptic patients low level of S-G-17prand (<10 pmol L $\mathrm{L}^{-1}$ ) and/or S-PGI $\left(<50 \mu \mathrm{g} \mathrm{L}^{-1}\right)$, were found in 16 of 19 patients $(84.2 \%)$ with MAG and in 7 of 36 patients $(19.4 \%)$ without atrophy in the histological study. 
They recommended that the test panel may be used as the "serological gastric biopsy" detecting MAG. The decrease in S-G-17and S-PGI as atrophic gastritis worsens is certainly based on a loss of normal mucosal glands and cells in the antrum and corpus mucosa and with the extension of IM especially in the antrum ${ }^{[9]}$. Several studies ${ }^{[18,25,26]}$ have shown that the Hp seropositivity rate was higher $(72 \%)$. Ley et $a l^{[26]}$ found of 205 subjects $87 \%$ had antibodies to $\mathrm{Hp}, 89 \%$ had histological evidence of $\mathrm{Hp}$ infection and $70 \%$ subjects had histological evidence of chronic atrophic gastritis. Among them the diagnosis was moderate and severe gastritis in $60 \%$. A high prevalence of moderate or severe chronic atrophic gastritis may be partially explained by the nearly universal infection with $\mathrm{Hp}^{[18,26]}$. Low S-G-17 $\left(<5 \mathrm{pmol} \mathrm{L}^{-1}\right)$ indicates atrophic antral gastritis only in subjects who are Hp-positive and have gastric inflammation ${ }^{[10]}$. In the study of Sipponen et $a l^{[10]}$ Vaananen et al. ${ }^{[13]}$ low S-G-17( $<5$ pmol L $\left.{ }^{-1}\right)$ and S-PGI $\left(<25 \mu \mathrm{g} \mathrm{L}^{-1}\right)$ occurred in $15 \%$ and in $10 \%$ of subjects with NAG respectively. In the present study low S-G-17 occurred also in 5 of $32(15.62 \%)$ subjects with NAG. Low S-G-17 does not indicate antral atrophy in these subjects. It is possible that these subjects are genuine hypersecretors of hydrochloric acid which inhibits the release of G-17 from antrum into the circulation. In the presence of $\mathrm{Hp}$, these subjects may be those who are particularly liable to antral atrophy and subsequently are also prone to peptic ulcer and reflux diseases ${ }^{[10.13]}$. We found that levels of S-G-17prand were significantly higher in corpus atrophy in comparison to those patients with antral atrophy. Similar finding was observed by ${ }^{[10]}$. An increase of serum gastrin also occurs in hypo- or achlorhydria: e.g. in the presence of atrophy in the corpus mucosa ${ }^{[27]}$. A long-lasting low acidity may even cause hyperplasia of antral $G$ cells. This occurs particularly in patients in whom the hypoacidity atrophic gastritis in the corpus occurs in connection with non-atrophic antrum ${ }^{[28]}$. If the antral mucosa is concomitantly atrophic (MAG), the S-G-17 level does not rise and the blood test panel shows both low S-PGI and S-G-17 values ${ }^{[29]}$. The presence of $\mathrm{Hp}$ infection and gastritis raised the S-G-17prand and S-PGI in subjects with $\mathrm{NAG}^{[30]}$. Elevating $\mathrm{G}$ cell numbers and gastrin secretion, in turn, stimulates parietal cells. As a result, the stomach is able to maximize its gastric acid output in an attempt to clear bacterial infection ${ }^{[31]}$. There are no significant variations in the chief cells in comparison with healthy controls in the case of NAG. The S-PGI increases significantly in the case of NAG as compared with healthy controls, decreases non-significantly in pre-atrophic gastritis and is significantly reduced in the case of atrophic gastritis ${ }^{[32]}$. Owing to the opposite influence of atrophy on S-G-17, the patients with Hprelated atrophic antral gastritis showed much lower $\mathrm{S}$ G-17 levels than the patients who were infected with
Hp but had no atrophy in the antrum. All 13 patients in the present study with an $\mathrm{Hp}$-related advanced atrophic antral gastritis had S-G-17 prand $<5$ pmol L $\mathrm{L}^{-1}$, as was the case also in the study of Sipponen et al. ${ }^{[10]}$. In the study of Vaananen et al. ${ }^{[13]}$ of the nine patients with an Hp-positive moderate or severe atrophic antral gastritis, six had S-G-17prand levels $<10$ pmol L ${ }^{-1}$. Of the 45 patients with moderate or severe corpus atrophy in endoscopic biopsies, 35 patients had S-PGI levels $<25$ $\mu \mathrm{g} \mathrm{L}^{-1}$. The present data showed that 4 of 6 patients had S-PGI $\left(<25 \mu \mathrm{g} \mathrm{L}^{-1}\right)$. We found that 7 of 15 patients with MAG had S-G-17prand and S-PGI $<5$ pmol L $\mathrm{L}^{-1}$ and $<25 \mu \mathrm{g} \mathrm{L}^{-1}$, meanwhile, the other 8 had these tests $<10 \mathrm{pmol} \mathrm{L}^{-1}$ and $<50 \mu \mathrm{g} \mathrm{L}^{-1}$ respectively. With the extension of gastric inflammation caused by $\mathrm{Hp}$ infection, gastric mucosal atrophy extends from the antrum to the corpus ${ }^{[25]}$. Sipponen et al. ${ }^{[10]}$ reported that among 4 patients with MAG, the S-G-17 prand tended to be $<5$ pmol L $\mathrm{L}^{-1}$ in 1 patient and $<10 \mathrm{pmol} \mathrm{L}^{-1}$ in 3 patients concomitantly showed low S-PGI (below $25 \mu \mathrm{g}$ $\mathrm{L}^{-1}$ in 3 of 4 patients). This indicates that a concomitant consideration of all three tests (Hp, S-G-17prand and SPGI) is necessary if the test values are to be interpreted properly.

In our patients endoscopy/histopathology revealed follicular gastritis in 10, gastric ulcer in 1 and erosion in 4. Previous studies ${ }^{[33,34]}$ reported that in Hp-positive gastric ulcer patients more atrophy of the antrum and the mean gastrin level was significantly lower than in Hp-positive individuals without peptic ulcer disease. Follicular gastritis was detected in our study in 10 patients ( 5 with moderate-severe antral atrophy, 1 with severe corpus atrophy, 4 with MAG; Hp was found in 9 of them verified both serologically and histopathologically. Low-grade mucosa-associated lymphoid tissue lymphomas arise from lymphatic tissue of the mucosa and were associated with chronic follicular gastritis, atrophy and IM. Hp was found in $75 \%$ of all lymphomas ${ }^{[25]}$.

The progression of $\mathrm{Hp}$-induced gastritis, to severe atrophic gastritis associated with metaplastic/ dysplastic changes, or necrotic tissues would possibly explain the absent or loss of $\mathrm{Hp}$ growing under these circumstances $^{35-37]}$. The disappearance of $\mathrm{Hp}$ has been documented in their studies during the progression of atrophic gastritis and in time serology is the only sign that indicates a previous infection. Twenty five $\%$ of patients were histologically and serologically negative, in $53 \% \mathrm{Hp}$ was not detected on histology but IgG to $\mathrm{Hp}$ were in all these patients elevated and $22 \%$ of patients were found to be positive at histology in the corpus mucosa; all but one of these patients had elevated circulating $\mathrm{IgG}$ to $\mathrm{Hp}^{[35]}$. Therefore, it is probable that $\mathrm{Hp}$ alone is not directly associated with stomach carcinogenesis. Instead, $\mathrm{Hp}$ appears to influence stomach carcinogenesis through the development of 
atrophic gastritis ${ }^{[23,35,36]}$. IM increases the risk of gastric cancer development. The overall prevalence of IM was higher in subjects with lower PGI values which could be used as a screening test for high-risk subjects with $\mathrm{IM}^{[37]}$. We encountered 8 patients with severe atrophic gastritis and IM/dysplasia, all of them had S-HpAb but only 6 of them had detected pathogen and S-G17 prand $<5 \mathrm{pmol}^{-1}$ and S-PG1 $<25 \mu \mathrm{g} \mathrm{L}^{-1}$. All of the 25 or 15 patients with atrophic changes in antrum or both the antrum and corpus had helicobacters verified by serology; meanwhile on histological examination one case was missed in each of them. These 2 patients had IM/dysplasia in addition to the atrophy. S-HpAb was not found in 3 cases with NAG meanwhile, histopathologically the pathogen was detected in them, the cause it may be an early infection in whom $\operatorname{IgA}$ might only be detected ${ }^{[38]}$. Oksanen et al ${ }^{[36]}$ mentioned that S-HpAb were strongly associated with atrophic antral gastritis but poorly associated with atrophic corpus gastritis and was $100 \%$ in both antrum and corpus. They found of the 27 patients with atrophic corpus gastritis, 16 patients had IM in the corpus. All 36 patients with atrophic antral gastritis had $\mathrm{IM}^{[36]}$.

The prevalence of dyspepsia is over $30 \%$ of global population; over half of the dyspeptic cases are functional dyspepsia. In these cases, the results from GastroPanel and gastroscopy indicate that the mucosa of the stomach is normal (no $\mathrm{Hp}$ infection, no inflammation, no atrophy ${ }^{[7.10,11,13]}$. In our study we encountered 8 subjects with functional dyspepsia. The most accurate diagnostic method of gastrointestinal tract diseases is endoscopy with subsequent biopsy. However, some histological sections could give falsenegative results because of patchy characteristics of atrophic changes in stomach mucosa ${ }^{[6,39]}$. The existing inflammation may also bias the microscopic appearance and lead to overestimation of atrophic gastritis due to artificial impression of a loss of glands ${ }^{[13]}$. Besides, biopsy is an expensive and labor-consuming method of research, so it could not be carried out for all patients in succession $^{[40]}$. For the selection of patients recommended to biopsy, the presence of a screening method is necessary. The serological testing of the gastric mucosa is of importance, given the fact that $\mathrm{Hp}$ eradication may cure gastritis and help to prevent further progression of gland loss that might also reduce the risk for gastric cancer ${ }^{[13,18,19,30,41,42]}$.

The panel is a good tool for non-endoscopic diagnosis and screening of atrophic gastritis ${ }^{[10,13,18,20]}$. The first two authors ${ }^{[10,13]}$ found that 82 and $96 \%$ respectively of their dyspeptic patients went into same 'gastritis categories' with both the S-G-17prand and SPGI and the endoscopy/histopathology by using the algorithm. In the present study, we found similar results in $92 \%$ of the patients. In our study S-G-17prand and SPGI showed a reasonable sensitivity, specificity, PPV, NPV and accuracy for diagnosis of atrophic gastritis.
Sipponen et al. ${ }^{[10]}$ found by using the cut-off levels for S-G-17prand and S-PGI with the best discrimination recommended in the algorithm, in delineation of patients with atrophic gastritis in general of any topographical type versus NAG and normal stomach the sensitivity, specificity, PPV, NPV and accuracy values of the blood test panel (S-G-17prand and S-PGI) were $89,93,70$ and $95 \%$ respectively. Meanwhile, by using only S-G-17prand $<10$ and $<5$ pmol $\mathrm{L}^{-1}$ : the sensitivity and specificity were 95,76 and 86 and $90 \%$ and by using only $\mathrm{S}-\mathrm{PGI}<25 \mu \mathrm{g} \mathrm{\textrm {L } ^ { - 1 }}$ : 84 and $95 \%$ respectively. Vaananen et al. ${ }^{[13]}$ found that the overall accuracy of the test panel as a biomarker of gastric mucosa was $83 \%$, the sensitivity, specificity, PPV and NPV were $83,95,75$ and $97 \%$. Ley et $a l^{[26]}$. reported that sensitivity and specificity for chronic atrophic gastritis for gastrin 83 and 22\% and for PGI 6 and 96\%. S-PGI levels may confirm the absence of chronic atrophic gastritis in a particular patient, but they are less useful in identifying persons at risk of this condition at the population level. The addition of PGI to gastrin improved specificities and positive predictive values, but dramatically decreased sensitivities ${ }^{[26]}$. We observed similar findings upon addition of S-G-17prand to SPGI.

The GastroPanel can be used as an initial method for screening and diagnosing patients suffering from dyspepsia, Hp-infection, atrophic gastritis and related risks and diseases ${ }^{[13]}$. The development of atrophic gastritis should be a strong indication for the gastroscopy and biopsy examinations as well as the eradication of $\mathrm{Hp}$. After the eradication of $\mathrm{Hp}$ the GastroPanel is an easer and much more sensitive method than the gastroscopy and biopsy examinations for following the healing of atrophic gastritis caused by Hp infection ${ }^{[13,40,42]}$.

Severe atrophic corpus gastritis was not determined by $\mathrm{Hp}$ tests but by low S-PGI and high S-G-17 ${ }^{[37]}$. The levels of S-G-17 are also influenced by the presence or absence of Hp gastritis in the stomach, by intragastric acidity and by various physiological stimuli. Therefore, in the consideration of S-G-17 as a biomarker of antral mucosa, the presence or absence of $\mathrm{Hp}$ gastritis and the grade of atrophy in the corpus mucosa (S-PGI) have to be taken into consideration simultaneously. This means that Hp, S-G-17 and S-PGI, have to be assayed concomitantly.

\section{CONCLUSION}

The serological gastric biopsy might be a feasible and appropriate diagnostic method for management of the dyspeptic patient. Detection of serum G-17 and PG1 levels can be offered as the screening tool for atrophic gastritis. However, the positive serologic results require further endoscopy with mucosal biopsy, for revealing probable progressing of atrophic process with development of peptic ulcer, IM/dysplasia or gastric cancer. Further studies of this serology will allow evaluation of the feasibility of screening and follow-up 
of treatment for gastritis and prevention of gastric cancer.

\section{REFERENCES}

1. Sipponen, P., 2001. Update on the pathologic approach to the diagnosis of gastritis, gastric atrophy and Helicobacter pylori and its sequelae. J. Clin. Gastroenterol., 32: 196-202.

2. International Agency for Research on Cancer, 1994. IARC monographs on the evaluation of carcinogenic risks to humans. Vol. 61: Schistosomes, liver flukes and Helicobacter pylori. Lyon: IARC.

3. Correa, P., 1992. Human gastric carcinogenesis: A multistep and multifactorial process. First American cancer society award lecture on cancer epidemiology and prevention. Cancer Res., 52: 6735-6740.

4. Malfertheiner, P., F. Megraud and C. O'Morain et al., 2002. Current concepts in the management of Helicobacter pylori infection-The Maastricht 2 Concensus Report. Aliment Pharmacol. Ther., 16: 167-180.

5. Ranta, P., T. Helske and I. Kaariainen et al., 2001. Serum pepsinogen I test reveals cases of vitamin B12 deficiency without anemia in patients with gastric corpus atrophy. Clin. Chem. Lab. Med., 39(suppl.): 328.

6. Rugge, M., P. Correa and M. Dixon et al., 2002. Gastric Mucosal Atrophy: interobserver consistency using new criteria for classification and grading. Aliment Pharmacol. Ther., 16: 124959.

7. Kokkola, A., H. Rautelin and P. Puolakkainen et al., 2000. Diagnosis of Helicobacter pyloriinfection in Patients with Atrophic Gastritis: Comparison of Histology, 13C. Urea Breath Test and Serology. Scand. J. Gastroenterol., 25: 138-41.

8. Yoshihara, M., K. Sumii and K. Haruma et al., 1998. Correlation of ratio of serum pepsinogen I and II with prevalence of gastric cancer and adenoma in Japanese subjects. Am. J. Gastroenterol., 93: 1090-96.

9. Stepan, V., K. Sugano, T. Yamada, J. Paerk and C. Dickson, 2002. Gastrin biosynthesis in canine $G$ cells. Am. J. Physiol. Gastrointest. Liver Physiol., 282: 766-75.

10. Sipponen, P., P. Ranta and T. Helske et al., 2002. Serum levels of amidated gastrin-17 and pepsinogen I in atrophic gastritis. An observational case-control study. Scand. J. Gastroenterol., 37: 785-91.

11. Nicolini, G., R. Zagari and P. Pozzato et al., 2002. Diagnosis of atrophic gastritis based upon a combination of three non-invasive tests: Preliminary results of the Loiano-Monghidoro project. J. Gastroenterol. Hepatol., 17(Suppl): A264 and Div Liver Dis. 2001; 33 Suppl.:A25.
12. Inoue, M., S. Kobayashi and A. Matsuura et al., 1998. Agreement of endoscopic findings and serum pepsinogen levels as an indicator of atrophic gastritis. Cancer Epidemiol. Biomark Prev., 7: 26163.

13. Vaananen, H., M. Vauhkonen and T. Helske et al., 2003. Non-endoscopic diagnosis of atrophic gastritis with a blood test. Correlation between Gastric Histology and Serum Levels of Gastrin-17 and Pepsinogen I. A Multicenter Study. Eur. J. Gastroenterol. Hepatol., 15: 885-91.

14. Dixon, M., R. Genta, J. Yardley, P. Correa and the participants of the International Workshop on the Histopathology of Gastritis, Houston 1996. Classification and grading of gastritis: the updated Sydney system. Am. J. Surg. Pathol., 20: 1161-81.

15. Hsu, S., L. Raine and H. Fanger, 1981. Use of avidin-biotin-peroxidase complex (ABC) in immunoperoxidase technique: A comparison between $\mathrm{ABC}$ and unlabelled antibody (PAP) procedures. J. Histochem. Cytochem., 29: 577.

16. Knight, T., J. Wyatt and A. Wilson et al., 1996. Helicobacter pylori gastritis and serum pepsinogen levels in a healthy population: Development of a biomarker strategy for gastric atrophy in high-risk groups. Br. J. Cancer, 73: 819-24.

17. Goetze, J. and J. Rehfeld, 2003. Impact of assay specificity in gastrinoma diagnosis. Clin. Chem., 49: 333-34.

18. Pasechnikov, V., S. Chukov and S. Kotelevets et al., 2004. Possibility of non-invasive diagnosis of gastric mucosal precancerous changes. World J. Gastroenterol., 10: 3146-50.

19. Sipponen, P., M. Harkonen, A. Alanko and O. Suovaniemi, 2002. Diagnosis of atrophic gastritis from a serum sample. Clin. Lab., 48: 505-15.

20. Wu, K., H. Li and T. Qiao et al., 2004. Diagnosis of atrophic body gastritis in Chinese patients by measuring serum pepsinogen. Chin. J. Dig. Dis., 5: 22-7.

21. Hartleb, M., P. Wandzel and M. Waluga et al., 2004. Non-endoscopic diagnosis of multifocal atrophic gastritis; efficacy of serum gastrin-17, pepsinogens and Helicobacter pylori antibodies. Acta Gastroenterol. Belg., 67: 320-26.

22. Saunders, D. and G. Trapp, 2001. Basic and Clinical Biostatistics. 3rd Edn. Connecticut, Appleton and Lang.

23. Cejas, H., A. Rodriguez and M. Herrero et al., 2001. Identification of Helicobacter pylori and associated gastric lesions. Rev. Fac. Cien. Med. Univ. Nac. Cordoba, 58: 65-76.

24. Bodger, K., J. Wyatt and R. Heatley, 2001. Variation in serum pepsinogens with severity and topography of Helicobacter pylori-associated chronic gastritis in dyspeptic patients referred for endoscopy. Helicobacter., 3: 216-24. 
25. Schlemper, R., S. van der Werf, I. Biemond and C. Lamers, 1996. Seroepidemiology of gastritis in Japanese and Dutch male employees with and without ulcer disease. Eur. J. Gastroenterol. Hepatol., 8: 33-9.

26. Ley, C., A. Mohar and J. Guarner et al., 2001. Screening markers for chronic atrophic gastritis in chiapas, Mexico. Cancer Epidemiol. Biomark Prev., 10: 107-12.

27. Valle, J., M. Kekki, P. Sipponen, T. Ihamiki and M. Siurala, 1996. Long term course and consequences of Helicobacter pylori gastritis. Results of a 32-year follow-up study. Scand. J. Gastroenterol., 31: 546-50.

28. Muller, J., T. Kirchner and H. Muller-Hermelink, 1987. Gastric endocrine cell hyperplasia and carcinoid tumors in atrophic gastritis type A. Am. J. Surg. Pathol., 11: 909-17.

29. Sipponen, P., J. Valle and K. Varis et al., 1990. Fasting levels of serum gastrin in different functional and morphologic states of the antrofundal mucosa. An analysis of 860 subjects. Scand. J. Gastroenterol., 25: 513-19.

30. Kalach, N., J. Legoedec and A. Wann et al., 2004. Serum levels of pepsinogen I, pepsinogen II and gastrin-17 in the course of helicobacter pylori gastritis in pediatrics. J. Pediatr. Gastroenterol. Nutr., 39: 568-70.

31. Zavros, Y., G. Rieder, A. Ferguson, L. Samuelson and J. Merchant, 2002. Genetic or chemical hypochlorhydria is associated with inflammation that modulates parietal and $\mathrm{G}$ cell populations in mice. Gastroenterol., 122: 119-34.

32. Perasso, A., G. Testino, P. de Angelis, C. Augeri and R. de Grandi, 1991. Gastric chief cell mass in chronic gastritis. Count and relationships to parietal cell mass and functional indices. Hepatogastroenterol., 38 (Suppl. 1): 63-6.

33. Ma, Z., T. Tanizawa, Z. Nihei, K. Sugihara and K. Nakamura, 2000. Follicular gastritis associated with Helicobacter pylori. J. Med. Dent. Sci., 47: 39-47.
34. Cheng, T., M. Tsou, M. Tsai and C. Chen, 2004. Early gastric MALT lymphoma. J. Chin. Med. Assoc., 67: 145-48.

35. Annibale, B., R. Negrini and P. Caruana et al., 2001. Two-thirds of atrophic body gastritis patients have evidence of Helicobacter pylori infection. Helicobacter., 6: 225-33.

36. Oksanen, A., P. Sipponen and R. Karttunen et al., 2000. Atrophic gastritis and Helicobacter pylori infection in outpatients referred for gastroscopy. Gut, 46: 460-63.

37. Urita, Y., K. Hike and N. Torii et al., 2004. Serum pepsinogens as a predicator of the topography of intestinal metaplasia in patients with atrophic gastritis. Dig. Dis. Sci., 49: 795-801.

38. Locatelli, A., W. Catapani, J. Gomes, C. Silva and J. Waisberg, 2004. Detection of anti-Helicobacter pylori antibodies in serum and duodenal fluid in peptic gastroduodenal disease. World J. Gastroenterol., 10: 2997-3000.

39. Moussa, A., B. Germana and S. Liatopoulou et al., 2004. Screening of gastric atrophy by gastropanel in primary care. Gut, 53(Suppl VI): A121.

40. Parsonnet, J., R. Harris, H. Hack and D. Owens, 1996. Modelling cost- effectiveness of Helicobacter pylori screening to prevent gastric cancer: a mandate for clinical trials. Lancet, 348:150.

41. Suovaniemi, O., M. Harkonen, L. Paloheimo and P. Sipponen, 2003. GastroPanel: diagnosing atrophic gastritis from serum - providing a tool for evidence-based medicine. Business Briefing:Global Health Care, 1-4.

42. Kuipers, E., 2003. In through the out door: Serology for atrophic gastritis. Eur. J. Gastroenterol. Hepatol., 15: 877-79. 